Revue des patrimoines

46 | 2022

Le patrimoine de la Justice

\title{
Le patrimoine de la Justice
}

\section{Marc Renneville et Michaël Vottero}

\section{OpenEdition}

Journals

Édition électronique

URL : https://journals.openedition.org/insitu/33898

DOI : $10.4000 /$ insitu.33898

ISSN : 1630-7305

\section{Éditeur}

Ministère de la Culture

\section{Référence électronique}

Marc Renneville et Michaël Vottero, « Le patrimoine de la Justice », In Situ [En ligne], 46 | 2022, mis en ligne le 16 janvier 2022, consulté le 03 février 2022. URL : http://journals.openedition.org/insitu/33898 ; DOI : https://doi.org/10.4000/insitu.33898

Ce document a été généré automatiquement le 3 février 2022

\section{(c) (i) (9)}

In Situ Revues des patrimoines est mis à disposition selon les termes de la licence Creative Commons Attribution - Pas d'Utilisation Commerciale - Pas de Modification 4.0 International. 


\title{
Le patrimoine de la Justice
}

\author{
Marc Renneville et Michaël Vottero
}

1 Le patrimoine de la Justice apparaît aujourd'hui encore peu connu et menacé. Les fermetures massives de tribunaux ont affecté les ensembles immobiliers, dispersé le mobilier de qualité et les collections ainsi que les bibliothèques et les archives. Les reconversions ont très souvent entrâné la disparition d'éléments de décor et d'aménagements intérieurs. De récentes mises aux normes, pour l'accessibilité et la sécurité, ont fait peser des risques de dénaturations d'espaces et d'aménagements anciens, allant jusqu'à la démolition de nombreuses prisons. Un certain nombre de protections au titre des monuments historiques ou de règlements d'urbanisme ont toutefois permis d'accompagner ces changements et certaines transformations, avec l'ajout d'éléments contemporains, apparaissent comme une nouvelle étape de l'histoire de la Justice en France et de l'évolution de ses pratiques'.

2 Depuis 1992 et l'ouvrage La Justice en ses temples², et un colloque organisé en 1994 par le Centre de recherche sur l'histoire de l'art et de l'architecture moderne (CRHAAM, CNRS / Paris IV) et l'Institut des hautes études sur la justice ${ }^{3}$ (IHEJ), peu de travaux ont été consacrés à cette thématique, en dehors de quelques monographies d'édifices ${ }^{4}$, d'un hors-série ancien de la revue Architecture intérieure. $C R E E^{5}$, et de la publication d'Étienne Madranges Les Palais de justice de France, en $2011^{6}$. Un récent numéro de la revue Monumental, consacré au patrimoine de l'enfermement, a permis de faire un point sur le corpus des bagnes et prisons ${ }^{7}$. En 2018 enfin, une recherche financée par le GIP ${ }^{8}$ «Mission de recherche. Droit et justice » a abouti à la mise en ligne de $\mathrm{HUGO}^{9}$, un site d'inventaire patrimonial collaboratif opéré par le Centre pour les humanités numériques et l'histoire de la justice (CLAMOR, CNRS / ministère de la Justice) et les Archives nationales ${ }^{10}$.

3 Près d'une centaine de palais de justice sont aujourd'hui protégés au titre des monuments historiques, dont certains anciennement, comme le palais de justice de Rouen, classé par liste dès $1840^{11}$. Aux côtés des constructions médiévales, renaissantes ou, pour le plus grand nombre, du XIX siècle, on trouve quelques créations du $\mathrm{xx}^{\mathrm{e}}$ siècle comme le palais de justice de Guadeloupe à Basse-Terre, de 1934, classé au titre des monuments historiques le 15 décembre 1997. De même, si l'on constate que les 
premières protections d'objets mobiliers dans des édifices de la Justice relèvent de périodes anciennes, avec la protection d'œuvres d'art précieuses comme des cartels, des fauteuils ou des tableaux, on assiste depuis quelques années à la protection d'ensembles du xix ${ }^{\mathrm{e}}$ siècle où figurent des bancs, des bureaux, des horloges et autres objets liés à l'activité de la Justice ${ }^{12}$. Un phénomène identique touche les lieux d'incarcération: les anciennes prisons médiévales ont été protégées avant celles du $\mathrm{XIX}^{\mathrm{e}}$ siècle $^{13}$. Ces protections découlent pour certaines des études réalisées par les services régionaux de l'Inventaire qui se sont intéressés aux édifices de la Justice, leur décor et leurs collections ${ }^{14}$. De même, des mémoires universitaires ont récemment étudié ce corpus ${ }^{15}$.

Outre l'architecture et les objets mobiliers, ce sont les archives qui font la richesse du patrimoine judiciaire. Qu'il s'agisse ici de documenter les édifices, leur construction ou leur évolution, les pratiques judiciaires d'instruction, de jugement ou d'exécution des peines, l'archive s'avère, une ressource indispensable. Le récolement des fonds, leur conservation, leur classement et leur mise à disposition participent ainsi pleinement d'une politique patrimoniale. Enfin, la valorisation du patrimoine judiciaire auprès du public pose bien des questions: reconstitution de vestiges ou restitution de traces disparues grâce au numérique mais aussi préservation d'édifices ou de matériels voués à la destruction, décision éminemment politique.

5 Signe positif d'un intérêt pour les thèmes concernés par le patrimoine de la Justice, l'appel à contributions lancé par la revue a suscité de nombreuses propositions qui témoignent de l'ampleur de ce sujet. Bien que représentations figurées de la Justice et des justiciables n'aient pas été traitées, les trois autres axes de réflexion proposés l'ont été abondamment, au point de nécessiter une publication en deux numéros, organisé chacun en deux axes.

6 Le premier numéro aborde le patrimoine architectural et les archives de la Justice. On s'intéresse ici à la fois aux questions d'inventaire, de politique patrimoniale mais aussi à l'évolution des perceptions d'une architecture « judiciaire » située au cœur de la cité, l'article d'Adrien Pitor évoquant ainsi les visites de l'enclos judiciaire à Paris aux XvII et XVIII ${ }^{\mathrm{e}}$ siècles. Plusieurs textes permettent de faire le point sur les études ou l'actualité récentes ayant trait à des édifices de la Justice, comme l'hôtel de Bourvallais à Paris, siège de la Chancellerie et actuel ministère de la Justice (Clémence Pau), le présidial de Limoges (Laure Leroux), le patrimoine de la Justice à Périgueux (Martine Balout) ou à Pau (Cécile Devos). Deux articles s'intéressent à des architectes spécialisés dans l'architecture judiciaire comme Pierre-Valentin Boudhors (Véronique Umbrecht) ou Guillaume Gillet (Franck Delorme). Le second axe s'attache à la documentation qui permet de faire l'histoire du patrimoine judiciaire en exposant, à partir de quelques exemples, la richesse et la diversité des fonds accessibles (imprimés, archives, cartes, plans...), leur exploitation mais aussi leurs lacunes, leurs limites et les nécessaires croisements de sources. Ces documents permettent de faire revivre des lieux disparus comme le bagne portuaire de Rochefort (Philippe Duprat), le siège ducal de Luynes, en Touraine (Patrick Bordeaux). Trois fonds d'archives sont présentés dans le numéro: celui de l'agence d'architecture du palais de justice de Paris (Nicolas Courtin), celui concernant les réalisations bordelaises conservé aux Archives nationales (Pascal Riviale et Clothilde Roullier), et les fonds d'action publique du ministère de la Justice (Manuel Toulajian). Enfin, certains lieux se spécialisent dans des thématiques judiciaires, à 
l'image du musée de Bretagne, à Rennes, et de ses collections liées à l'affaire Dreyfus (Laurence Prod'homme).

\section{NOTES}

1. Sur les récentes restaurations et réhabilitations de palais de justice, nous renvoyons au site de l'Agence publique pour l'immobilier de la Justice qui permet d'accéder aux projets par une carte de France: https://www.apij.justice.fr/nos-projets/la-carte-des-projets/ [lien valide en janvier 2022]. Le site permet également de télécharger des plaquettes de présentation des travaux réalisés, à l'image du palais de justice de Chalon-sur-Saône (Saône-et-Loire) : https:// www.apij.justice.fr/nos-projets/les-operations-judiciaires/palais-de-justice-de-chalon-sursaone/ [lien valide en janvier 2022].

2. ASSOCIATION POUR L'HISTOIRE DE LA JUSTICE, La Justice en ses temples. Regards sur l'architecture judiciaire en France, Paris / Poitiers, Errance / Brissaud, 1992.

3. «Les Palais de justice ", Monuments historiques, janvier-février 1996, $n^{\circ} 200$. Ce numéro reprend une partie des entretiens du colloque de 1994, dont les actes n'ont pas été publiés.

4. Sans être exhaustifs, nous pouvons citer parmi les récentes publications : Le Palais de Justice, catalogue d'exposition (Paris, Conciergerie), Paris, Action artistique de la Ville de Paris, 2002 ; IRVOAS-DANTEC \& BOHUON Philippe, Le Parlement de Bretagne. Rennes, Paris, Éditions du patrimoine, coll. «Itinéraires », 2005 ; BOYER Pierre-Louis \& BÉZY Jacques, Le Palais de justice de Toulouse. Un autre regard, Souyri, Au fil du temps, coll. «Architecture », 2013; DE BAECQUE Christian, DE MONCAN Patrice \& D'ARJUZON Jacques (dir.), 150 ans d'histoire derrière ces murs. Le Tribunal de Commerce de Paris, Paris, Éditions du Mécène, 2015.

5. « Construire pour la justice », Architecture intérieure. CREE, mai-juin 1995, n 265.

6. MADRANGES Étienne, Les Palais de justice de France. Architecture, symboles, mobilier, beautés et curiosités, Paris, Lexis Nexis, 2011.

7. «Le patrimoine de l'enfermement », Monumental, 2018-1.

8. Groupement d'intérêt public.

9. Voir https://hugo.criminocorpus.org/fr/ [lien valide en janvier 2022].

10. RENNEVILLE Marc (dir.), "HUGO. Patrimoine des lieux de justice », rapport de recherche, 2018 https://dumas.ccsd.cnrs.fr/GIP-MRDJ/halshs-01960688 [lien valide en janvier 2022].

11. La base Mérimée contient 97 notices de palais de justice protégés au titre des monuments historiques: https://www.pop.culture.gouv.fr/gallery/61b4ab2d14d0cc0e3d226ab0 [lien valide en janvier 2022].

12. La base Palissy contient ainsi 483 notices d'objets protégés au titre des monuments historiques conservés dans un palais de justice.

13. Ce sont 216 prisons ou anciennes prisons qui sont actuellement protégées au titre des monuments historiques.

14. La base Mérimée, consacrée au patrimoine architectural, contient 190 notices de palais de justice rédigées par les services régionaux de l'Inventaire, et la base Palissy, pour les objets mobiliers, 177 notices.

15. MOULIN Laure-Estelle, «L'Architecture judiciaire en France sous la V République », thèse de doctorat d'histoire de l'art (Paris, université Paris-I, 2006); HUGEDÉ Frédéric Christian, D'une image au service du public. Évolution des tribunaux de grande instance entre 1958 et 1987, mémoire MES, 
Villeneuve-d'Ascq, ENSAP de Lille, 2007 ; BELS Marie, «Les Grands Projets de la justice française. Stratégies et réalisations architecturales du ministère de la Justice (1991-2001) ", thèse de doctorat d'architecture, Champs-sur-Marne, université Paris-Est, 2013.

\section{AUTEURS}

\section{MARC RENNEVILLE}

DR CNRS. Centre Alexandre-Koyré ; Histoire des sciences et des techniques Directeur du Centre pour les humanités numériques et l'histoire de la Justice CLAMOR UAR 3726 CNRS - ministère de la Justice

\section{MICHAËL VOTTERO}

Conservateur régional adjoint des monuments historiques

DRAC Bourgogne-Franche-Comté

Chercheur associé du Centre Georges-Chevrier

Université de Bourgogne (UMR7366) 